Article

\title{
Analysis of Low Probability of Detection Capability for Chaotic Standard Map-Based FH-OFDMA System
}

\author{
Junwoo Jung ${ }^{1, * \mathbb{C}}$, Jaesung Lim ${ }^{2}$, Sungyeol Park ${ }^{1}$, Haengik Kang ${ }^{1}$ and Seungbok Kwon ${ }^{1}$ \\ 1 LIG Nex1, Sampyeong-dong, Bundang-gu, Sungnam 13488, Korea; syeol.park@lignex1.com (S.P.); \\ haengik.kang@lignex1.com (H.K.); seungbokkwon@lignex1.com (S.K.) \\ 2 Department of Computer Engineering, Ajou Univerity, San 5, Wonchon-dong, Youngtong-gu, \\ Suwon 16499, Korea; jaslim@ajou.ac.kr \\ * Correspondence: junu.jung@gmail.com; Tel.: +82-31-8026-4598
}

check for updates

Citation: Jung, J.; Lim, J.; Park, S.; Kang, H.; Kwon, S. Analysis of Low Probability of Detection Capability for Chaotic Standard Map-Based FH-OFDMA System. Appl. Sci. 2021, 11, 2198. https://doi.org/10.3390/ app11052198

Academic Editor: Christos Bouras

Received: 9 February 2021

Accepted: 26 February 2021

Published: 3 March 2021

Publisher's Note: MDPI stays neutral with regard to jurisdictional claims in published maps and institutional affiliations.

Copyright: (c) 2021 by the authors. Licensee MDPI, Basel, Switzerland. This article is an open access article distributed under the terms and conditions of the Creative Commons Attribution (CC BY) license (https:/ / creativecommons.org/licenses/by/ $4.0 /)$.

\begin{abstract}
A frequency hopping orthogonal frequency division multiple access (FH-OFDMA) can provide low probability of detection (LPD) and anti-jamming capabilities to users against adversary detectors. To obtain an extreme LPD capability that cannot be provided by the basic symbol-bysymbol (SBS)-based FH pattern, we proposed two FH patterns, namely chaotic standard map (CSM) and cat map for FH-OFDMA systems. In our previous work, through analysis of complexity to regenerate the transmitted symbol sequence, at the point of adversary detectors, we found that the CSM had a lower probability of intercept than the cat map and SBS. It is possible when a detector already knows symbol and frame structures, and the detector has been synchronized to the FHOFDMA system. Unlike the previous work, here, we analyze whether the CSM provides greater LPD capability than the cat map and SBS by detection probability using spectrum sensing technique. We analyze the detection probability of the CSM and provide detection probabilities of the cat map and SBS compared to the CSM. Based on our analysis of the detection probability and numerical results, it is evident that the CSM provides greater LPD capability than both the cat map and SBS-based FH-OFDMA systems.
\end{abstract}

Keywords: FH-OFDMA; frequency hopping; LPD; chaotic standard map

\section{Introduction}

Frequency hopping-orthogonal frequency division multiple access (FH-OFDMA) can provide low probability of detection (LPD) and anti-jamming capabilities to authenticated users against adversaries for secure multimedia and military communication services [1]. In the OFDMA system, the entire frequency spectrum is divided into multiple narrow-band subcarriers, which are then assigned to multiple users to transmit data symbols on different subcarriers simultaneously [2]. In the FH-OFDMA system, the total number of subcarriers is partitioned into a set of clusters composed of contiguous subcarriers, and each cluster is changed to a different frequency during each period based on the FH pattern. The users are each assigned to one of the clusters to transmit data symbols over the FH-OFDMA system. Thus, the FH-OFDMA system has the advantages of both LPD due to FH pattern and high spectral efficiency of the OFDMA system [3]. The FH-OFDMA system chooses the orthogonal frequency division multiplexing (OFDM) symbols and cyclic prefix durations according to the Doppler and delay spreads of the transmitting channel, respectively; thus, subcarriers of the FH-OFDMA system retain their orthogonality and experience flat fading upon reception. The FH-OFDMA system can be combined with channel coding resulting in frequency diversity when the channel response varies over the hopping distance, and such frequency diversity has been verified in many prior studies [4-6]. In the military domain, the authors of Reference [7] consider the condition that an adversary intentionally jams parts of frequency hopping slots and select non-jammed hopping sequences to obtain low bit error performance. However, in this study, we have fundamentally different focus on the 
advantage of the FH-OFDMA system that supports LPD against detection of adversaries, such as eavesdroppers or radiometers.

Some previous works on FH-OFDMA systems have noted various FH pattern designs to achieve LPD capability and frequency diversity [8-13]. In Reference [8-12], the transmitted symbol index is changed at each symbol period through the symbol-by-symbol (SBS) hopping strategy. In order to design an SBS-based FH pattern, orthogonal Latin squares and Welch-Costas-based FH patterns are introduced in Reference [8,9], respectively. In Reference $[10,11]$, secure collision free and residue number system arithmetic assisted FH patterns are proposed to obtain high frequency diversity through SBS hopping over fast frequency selective fading. In [12], the authors propose a wide-gap $\mathrm{FH}$ pattern, which can be adopted to FH-OFDMA systems, to highly reduce interference between adjacent frequencies. In [13], we propose two frequency hopping strategies based on the chaotic standard map (CSM) and cat map-based FH patterns. As these two FH patterns are hopped into time and frequency domains using additional memory of the cluster size, the FHOFDMA systems of these two proposed FH patterns have lower probabilities of intercept than the SBS-based FH pattern. Between the CSM and cat map-based FH patterns, the CSM-based FH-OFDMA system has a lower probability of intercept, since a symbol of the CSM cannot be shown in every symbol period, whereas a symbol of the cat map is mixed with the symbol index and is shown in every symbol period similar to the SBS. In Reference [13], we only analyze the complexity of regenerating the transmitted symbol sequence. It is possible that a detector already knows the symbol and frame structures, and their parameters, and the detector is synchronized to the FH-OFDMA system.

Unlike our previous work, here, we analyze whether the CSM provides greater LPD capability than the cat map and SBS-based FH-OFDMA systems by detection probability using spectrum sensing technique. The spectrum sensing technique is studied to identify unused spectrum regions that may be exploited efficiently without causing harmful interference to the existing incumbent users. Although it is very difficult to detect authentic OFDMA signals using spectrum sensing, many studies related to cognitive radio techniques have helped the spectrum sensing domain [14]. Based on spectrum sensing, the authors of Reference [15] propose an FH pattern based on SBS hopping strategy for LPD capability, and the authors analyze detection probability in frequency-hopped wireless communications. Up until very recently, many authors have proposed the signal sensing methods to obtain FH signals based on cross-correlation [16] and deep learning [17]. These detection methods are only focused on the frequency-hopped system, but do not consider an OFDM system constructed by multiple subcarriers. For the OFDM system, an auto-correlation-based spectrum sensing technique is proposed to analyze the detection probability in Reference [18]. Although the auto-correlation-based OFDM signal sensing technique does not require knowledge of the noise variance, the sensing technique for the FH-OFDMA system has different conditions for detection probabilities because of regenerated hopping sequence. We assume that the detector exploits the auto-correlation-based spectrum sensing technique with hypothesis testing to detect the FH-OFDMA signal. We analyze detection probability of the CSM-based FH-OFDMA system and provide detection probabilities of the cat map and SBS-based FH-OFDMA systems compared to the CSM. Based on our analysis of the detection probability, it is evident that the CSM provides a higher LPD capability than both the cat map and SBS-based systems.

The rest of this paper is organized as follows: In Section 2, our CSM and cat mapbased FH patterns proposed in Reference [13] are introduced, along with the SBS-based FH pattern for FH-OFDMA systems. In Section 3, we analyze the detection probability based on auto-correlation properties of OFDMA signals with hypothesis testing for the FH-OFDMA systems. Numerical simulations of the systems with specific parameters are carried out, and the simulation results are shown in Section 4. Finally, our conclusions are presented in Section 5. 


\section{FH Patterns for FH-OFDMA System}

The total bandwidth is divided into a number of subcarriers $N_{F F T}$ according to the length of the fast Fourier transform (FFT) signal of the FH-OFDMA system, which is composed of contiguous OFDMA symbols consisting of a sum of subcarriers modulated by multiple phase shift keying (PSK) or quadrature amplitude modulation (QAM). Without loss of generality, we assume the sampling rate between the number of effective symbols $T_{d}$, and the number of subcarriers $N_{F F T}$, after the inverse FFT (IFFT) as 1 . The size of each subcarrier is constructed using several clusters with the number of clusters $N_{L}$, which are composed of contiguous subcarriers whose number is denoted as $N_{S}$. Thus, we achieve $N_{L}=N_{F F T} / N_{s}$. The FH-OFDMA signal converts from serial to parallel by an IFFT block. Let $D_{n}(0), D_{n}(1), \ldots, D_{n}\left(N_{F F T}-1\right)$ be complex multiple PSK or QAM modulated data that are transmitted on $N_{F F T}$ subcarriers at the $n$-th cluster index; then, the output of the IFFT as the symbol of the transmitted FH-OFDMA frame is

$$
s_{n}(t)=\frac{1}{\sqrt{N_{F F T}}} \sum_{f=0}^{N_{F F T}-1} D_{n}(f) e^{\frac{j 2 \pi t f}{N_{F F T}}},
$$

where $t$ is a discrete time index, $t=0, \ldots, T_{d}-1, n$ is cluster index from 0 to $N_{L}-1$, and $f$ is a discrete subcarrier index. Let $T_{c}$ be the number of symbols for the cyclic prefix; we define cyclic prefix symbols of the $n$-th cluster as $s_{n}\left(T_{d}-T_{c}\right), \ldots, s_{n}\left(T_{d}-1\right)$, and the cyclic prefix symbols are added to the FH-OFDMA symbols. In the time domain, the FH-OFDMA time block with duration $T_{s}=T_{d}+T_{c}$ is constructed as $\left[s_{n}\left(T_{d}-T_{c}\right), \ldots, s_{n}\left(T_{d}-1\right), s_{n}(0), s_{n}(1)\right.$, $\left.\ldots, s_{n}\left(T_{d}-1\right)\right]$.

In the FH-OFDMA system, the modulated data with $N_{F F T}$ size before an IFFT block are constructed by a specific $\mathrm{FH}$ pattern given by

$$
\sum_{f=0}^{N_{F F T}-1} D_{n}(f)=\left\{\sum_{k} \sum_{i=0}^{N_{L}-1} \sum_{t=i \times N_{s}}^{i \times N_{s}+\left(N_{s}-1\right)} d_{n}^{k}(t) \mid k \in\left\{H_{\alpha, x}^{k}(j)=i, H_{\alpha, y}^{k}(j)=n\right\}\right\},
$$

where $d_{n}^{k}(t)$ denotes the transmitted data of $k$-th user for $n$-th cluster at $t$-th symbol time index. $H_{\alpha, x}^{k}(j)$ and $H_{\alpha, y}^{k}(j)$ are the $x$-axis and $y$-axis of the FH pattern for the $j$-th generated data of $k$-th user, respectively. Each user generates data of size $N_{L}$, and the data is mixed and modulated by the FH pattern, such as CSM, cat map, and SBS-based FH pattern. $x$-axis and $y$-axis indicate the range of the symbol and cluster indexes, respectively. Since we consider that a user can be assigned only one cluster index in the FH-OFDMA system, the maximum number of users is limited to $N_{L}$. With a seed number of $\alpha$, we assign the value of $\left\{H_{\alpha, x}^{k}(j), H_{\alpha, y}^{k}(j)\right\}$ to $\left\{L_{\alpha, x}^{k}(j), L_{\alpha, y}^{k}(j)\right\},\left\{T_{\alpha, x}^{k}(j), T_{\alpha, y}^{k}(j)\right\}$, and $\left\{C_{\alpha, x}^{k}(j), C_{\alpha, y}^{k}(j)\right\}$ for the SBS, cat map, and CSM-based hopping strategies, respectively. In Figure 1, we set $N_{s}=4$ and show the symbol of a transmitted FH-OFDMA frame as an example. The same-colored blocks are transmitted contiguously by the same user. As shown in Figure 1, the number of symbols for cyclic prefix, $T_{\mathcal{c}}$, is copied from the number of effective symbols, $T_{d}$, and is added in front of the effective symbols to absorb the multipath delay of the FH-OFDMA system. 


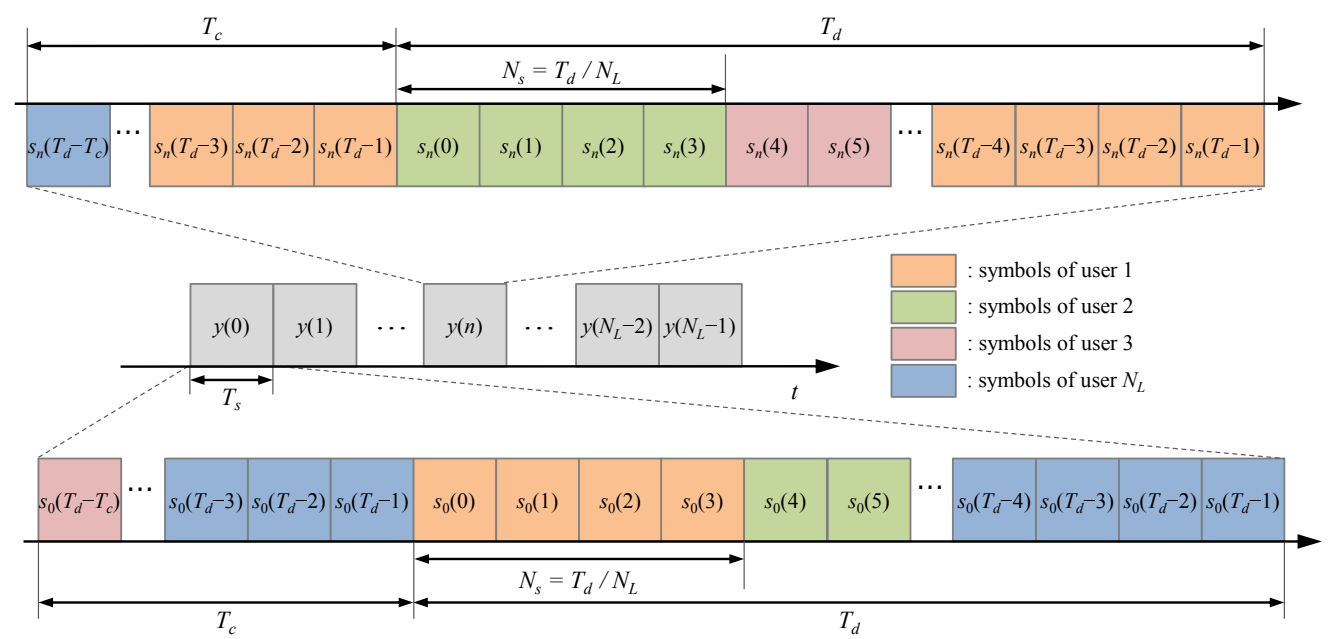

Figure 1. Contiguous frequency hopping orthogonal frequency division multiplexing (FH-OFDM) symbol structure for spectrum sensing.

Before we introduce three FH patterns to verify the LPD capabilities of the FH-OFDMA system, we address that the CSM and cat map-based FH patterns have practical limitations that those FH patterns must have symbol buffers with size $N_{L}$ unlike the SBS-based FH pattern. Because the transmitting data sequences of the two FH patterns are mixed to increase the complexity of detection probability against eavesdroppers. In addition, receivers of the FH-OFDMA systems using the CSM and cat map-based FH patterns can start parsing modulated data after receiving $N_{L}$ symbol buffers. Now, we show the SBSbased FH pattern and its FH pattern indexes of $x$-axis and $y$-axis. In this study, we choose a formula of the Latin squares-based FH pattern as one of the SBS-based FH patterns [13]. We can obtain the SBS-based FH pattern of the $j$-th data for the $k$-th user using Latin squares as

$$
\left\{\begin{array}{l}
L_{\alpha, x}^{k}(j)=j \\
L_{\alpha, y}^{k}(j)=\left((k-j) \alpha^{-1}\right) \bmod N_{L}
\end{array},\right.
$$

where $\alpha$ is the seed number of the FH pattern for $\alpha=2, \ldots, N_{L}-1$. We introduce the cat map-based FH pattern using block symmetric ciphers [13]. In the cat map-based FH pattern, the generated symbols appear to be hopped in both time and frequency domains, since the transmitted symbol of each user appears at each period once, but the transmission order is mixed. The cat map-based FH pattern of the $j$-th data for the $k$-th user can be obtained by

$$
\left[\begin{array}{c}
T_{\alpha, x}^{k}(j) \\
T_{\alpha, y}^{k}(j)
\end{array}\right]=\left[\begin{array}{ll}
b_{11} & b_{12} \\
b_{21} & b_{22}
\end{array}\right]\left[\begin{array}{c}
c_{\alpha}^{k}(j) \\
j
\end{array}\right]\left(\bmod N_{L}\right)
$$

where $b_{11}, b_{12}, b_{21}$, and $b_{22}$ are positive integers satisfying relations $b_{11} \times b_{22}-b_{12} \times b_{21}=1$ and $\alpha b_{12}>b_{11}$. The cluster index of $c_{\alpha}^{k}(j)$ of the $j$-th data for the $k$-th user using a seed number of $\alpha$ is given by

$$
c_{\alpha}^{k}(j)=\left((k-j) \alpha^{-1}\right) \bmod N_{L}, j=0, \ldots, N_{L}-1,
$$

where $j$ denotes the data index of consecutive points $(i, j)$ for which $L_{\alpha}(i, j)=k$ and $L_{\alpha}(i, j)=(\alpha i+j) \bmod N_{L}, i, j=0, \ldots, N_{L}-1$ [13]. The CSM is widely used to design block symmetric ciphers for LPD capability. Beside the cat map-based FH pattern, the symbols generated by the CSM-based FH pattern are hopped in both time and frequency domains, and the transmitted sequences are mixed. Based on the complexity of detection probability, the CSM-based FH pattern provides greater LPD capability than the cat map and SBS-based FH patterns. The CSM-based FH pattern of the $j$-th data for the $k$-th user can be obtained by 


$$
\left\{\begin{array}{l}
C_{\alpha, x}^{k}(j)=\left(c_{\alpha}^{k}(j)+j+r_{x}+r_{y}\right) \bmod N_{L} \\
C_{\alpha, y}^{k}(j)=\left(j+r_{y}+\left[K_{C} \sin \frac{C_{\alpha, x}^{k}(j) N_{L}}{2 \pi}\right]\right) \bmod N_{L}{ }^{\prime}
\end{array}\right.
$$

where the cluster index of $c_{\alpha}^{k}(j)$ of the $j$-th data for the $k$-th user using a seed number $\alpha$ can be obtained by (5). $r_{x}$ and $r_{y}$ are constant integers from 0 to $N_{L}-1$, and $K_{C}$ is a positive integer. $[x]$ is the nearest integer to $x$.

Figure 2 shows an example of how to assign the three $\mathrm{FH}$ patterns in time and subcarrier domains, and we compare the three FH patterns, i.e., the SBS, cat map, and CSM-based patterns. To increase the detection probability from the point of view of eavesdropping detectors, we assume that the detectors receive a redundant number, $N_{s}$, of contiguous FH-OFDMA frames consisting of $N_{L}$ symbols. For the examples in Figure 2, we set $N_{L}=5, N_{F F T}=20, N_{S}=4$, number of transmitting users as 3, and indexes of the users as $k=1,2$, and 5. In Figure 2a, we show an example of an SBS-based FH pattern using Latin squares-based strategy for seed number, $\alpha=4$. The users, $k=1,2$, and 5 are assigned their SBS-based FH patterns as $\left\{L_{4, x}^{1}(j), L_{4, y}^{1}(j)\right\}=\{(1,1),(2,2),(3,3),(4,4),(5,5)\}$, $\left\{L_{4, x}^{2}(j), L_{4, y}^{2}(j)\right\}=\{(5,5),(1,1),(2,2),(3,3),(4,4)\}$, and $\left\{L_{4, x}^{5}(j), L_{4, y}^{5}(j)\right\}=\{(2,2),(3,3)$, $(4,4),(5,5),(1,1)\}$ for $j=1, \ldots, 5$, respectively. As shown in Figure $2 \mathrm{a}$ as an example of the SBS-based FH pattern, the data index of each user is shown once at each cluster and are hopped to a different cluster for each symbol. In addition, the transmitting data order of every user is continuous in Figure 2a. In Figure 2b, we show an example of a cat map-based FH pattern for $\left\{b_{11}, b_{12}, b_{21}, b_{22}\right\}=\{1,1,1,2\}$ and seed number, $\alpha=4$. The users, $k=1,2$, and 5 are assigned their cat map-based FH patterns as $\left\{T_{4, x}^{1}(j), T_{4, y}^{1}(j)\right\}=$ $\{(1,1),(3,4),(5,2),(2,5),(4,3)\},\left\{T_{4, x}^{2}(j), T_{4, y}^{2}(j)\right\}=\{(5,5),(2,3),(4,1),(1,4),(3,2)\}$, and $\left\{T_{4, x}^{5}(j), T_{4, y}^{5}(j)\right\}=\{(2,2),(3,3),(4,4),(5,5),(1,1)\}$ for $j=1, \ldots, 5$, respectively. As shown in Figure $2 \mathrm{~b}$ as an example of the cat map-based FH pattern, the first data set for $k=2$ is transmitted at the symbol period of $n=4$ and the first data set for $k=5$ is transmitted at the symbol period of $n=1$. In the cat map-based FH pattern, the transmitted data order is mixed as shown in Figure $2 b$. However, the data index of each user using the cat map-based FH pattern is shown once for each cluster and hopped to a different cluster for each symbol. In Figure 2c, we show the example of a CSM-based FH pattern for $K_{C}=3000, r_{x}=1, r_{y}=3$ and seed number $\alpha=2$. The users, $k=1,2$, and 5 are assigned their CSM-based FH patterns as $\left\{C_{4, x}^{1}(j), C_{4, y}^{1}(j)\right\}=\{(5,5),(3,4),(1,1),(4,1),(2,1)\}$, $\left\{C_{4, x}^{2}(j), C_{4, y}^{2}(j)\right\}=\{(3,3),(1,5),(4,5),(2,5),(5,4)\}$, and $\left\{C_{4, x}^{5}(j), C_{4, y}^{5}(j)\right\}=\{(2,2),(5,1)$, $(3,5),(1,2),(4,2)\}$ for $j=1, \ldots, 5$, respectively. As shown in Figure $2 \mathrm{c}$ as example of the CSM-based FH pattern, two data sets for $k=1$ appeared at the symbol period $n=1$, and two data sets for $k=5$ appeared at the symbol period $n=2$. In addition, the transmitted data order is mixed, since the last and first data sets for $k=1$ is transmitted at the symbol periods $n=0$ and $n=4$, respectively. 


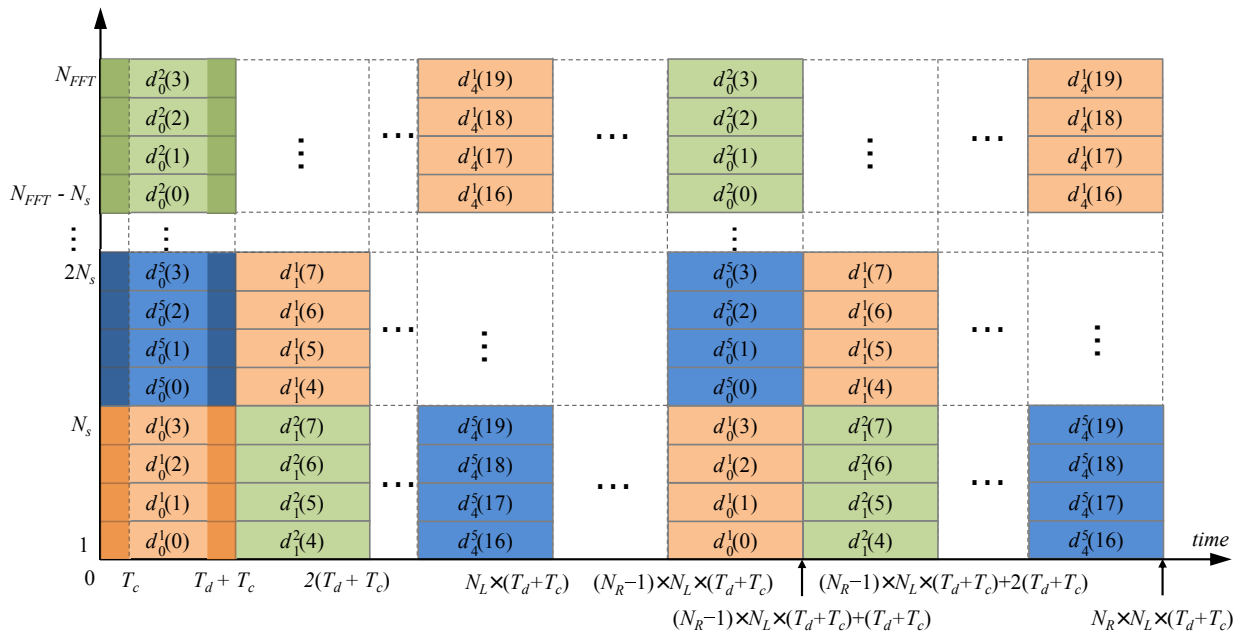

(a) Example of an SBS-based FH pattern

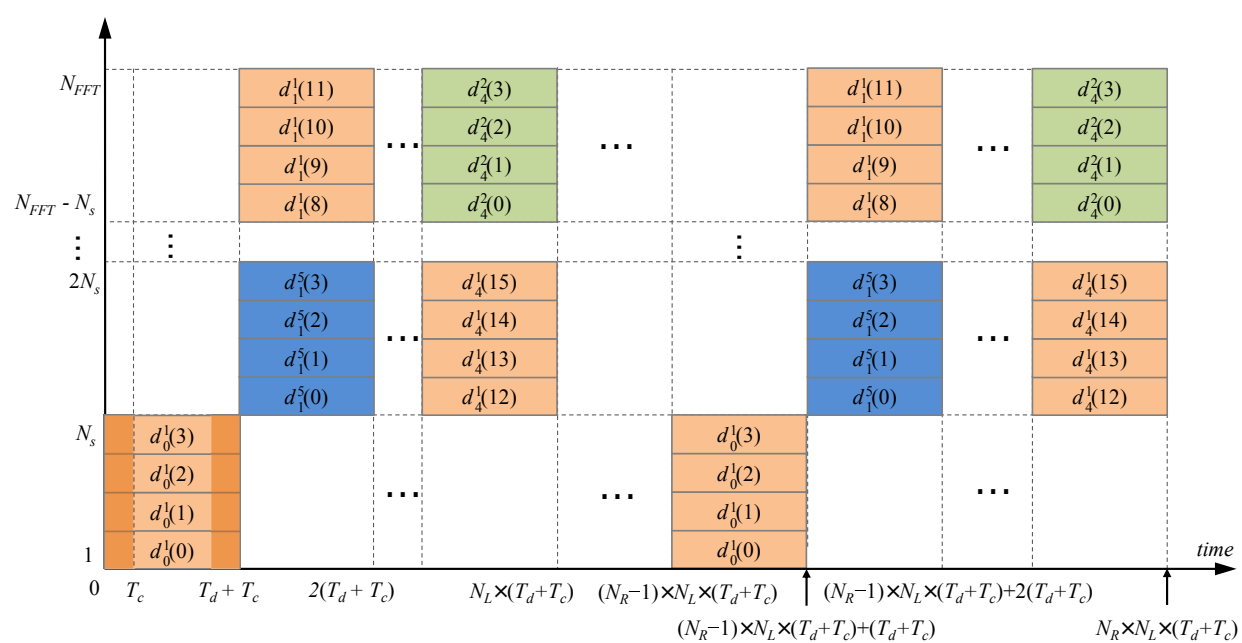

(b) Example of a cat map-based FH pattern

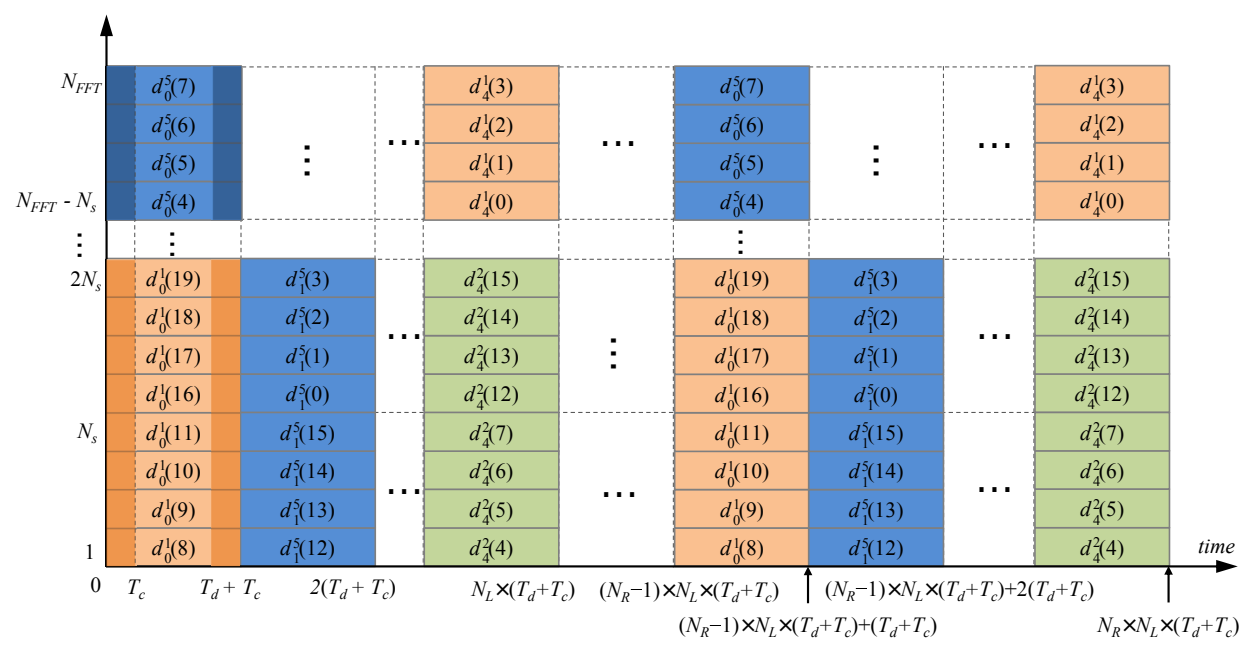

(c) Example of a CSM-based FH pattern

Figure 2. Comparison of symbol-by-symbol (SBS), cat map, and chaotic standard map (CSM)-based FH patterns. 


\section{Analysis of Detection Probability}

In this section, our goal is to analyze detection probability of a CSM-based FH-OFDMA system compared to detection probabilities of the other FH-OFDMA systems from the point of view of an adversary detector. To increase detection probability, the detector should observe over several FH-OFDMA symbols, i.e., $y(0), y(1), \ldots, y\left(M+T_{d}-1\right)$. The number of observing FH-OFDMA symbols, $M$, must be composed of multiplications of the redundant number of FH-OFDMA frames, $N_{R}$, and the number of symbols in a frame, i.e., $M=N_{R} \times N_{L}$. Our analysis assumes that the adversary detector already knows the effective symbol duration $T_{d}$ and its cyclic prefix $T_{c}$. The knowledge of symbol duration is specified in standards or can be estimated by knowledge of the implementable IFFT and FFT sizes. Even if the exact value is not known, the symbol duration can be estimated by the receipt of sufficient symbols.

We analyze the detection probability using the spectrum sensing technique based on hypothesis testing. First, let $h_{0}$ be the null hypothesis, i.e., a transmitting user is absent in a FH-OFDMA system, and $h_{1}$ be the alternate hypothesis, i.e., a transmitting user exists in the FH-OFDMA system. Thus, the hypothesis testing problem may be written as

$$
\left\{\begin{array}{l}
h_{0}: y(t)=w(t) \\
h_{1}: y(t)=s(t)+w(t)
\end{array},\right.
$$

where $y(t)$ is the received complex FH-OFDMA signal, and $w(t)$ is the complex circular additive white Gaussian noise. Invoking the central limit theorem under the assumption of sufficiently large IFFT size, we have

$$
\text { and } \begin{aligned}
s(t) & \sim \mathcal{N}_{c}\left(0, \sigma_{s}^{2}\right) \\
w(t) & \sim \mathcal{N}_{c}\left(0, \sigma_{w}^{2}\right)
\end{aligned},
$$

where $\mathcal{N}_{c}($.$) denotes the Gaussian distribution for a complex random variable. Therefore,$ for different hypotheses, we have

$$
\left\{\begin{array}{l}
h_{0}: y(t) \sim \mathcal{N}_{c}\left(0, \sigma_{w}^{2}\right) \\
h_{1}: y(t) \sim \mathcal{N}_{c}\left(0, \sigma_{s}^{2}+\sigma_{w}^{2}\right) .
\end{array} .\right.
$$

We consider $y(t)=y_{r}(t)+j y_{i}(t)$ as a circularly symmetric Gaussian random variable composed of real and imaginary parts of $y$, and these real and imaginary parts are obtained as:

$$
\text { and } \begin{aligned}
y_{r}(t) & \sim \mathcal{N}_{r}\left(0, \sigma_{y}^{2} / 2\right) \\
y_{i}(t) & \sim \mathcal{N}_{r}\left(0, \sigma_{y}^{2} / 2\right)
\end{aligned},
$$

where $\mathcal{N}_{r}(\cdot)$ denotes the Gaussian distribution for a real random variable. For the $\mathrm{FH}-$ OFDMA signal using cyclic prefix, the values of the auto-correlation coefficient $\rho(\tau)=E\left[y(t) y^{*}(t+\tau)\right] / E\left[y(t) y^{*}(t)\right]$ for delay $\tau= \pm T_{d}$ under the two hypotheses are

$$
\left\{\begin{array}{l}
h_{0}: \rho\left( \pm T_{d}\right)=0 \\
h_{1}: \rho\left( \pm T_{d}\right)=\rho_{D}
\end{array},\right.
$$

where

$$
\begin{aligned}
\rho_{D} & =\frac{T_{c}}{T_{d}+T_{c}} \frac{\sigma_{s}^{2}}{\sigma_{s}^{2}+\sigma_{w}^{2}} \\
& =\frac{T_{c}}{T_{d}+T_{c}} \frac{\Gamma}{1+\Gamma}
\end{aligned}
$$

where $\Gamma$ denotes the signal-to-noise ratio (SNR) as $\sigma_{s}^{2} / \sigma_{w}^{2}$ and $0<\rho_{D}<1$. For a real valued coefficient, $\rho(\tau)=\rho(-\tau)$. Hence, without loss of generality, we consider only $\tau=T_{d}$.

We can form two real random vectors from the observations such that $\mathbf{z}_{1}=\left[y_{r}(0) y_{i}(0)\right.$ $\left.y_{r}(1) y_{i}(1) \ldots y_{r}(M-1) y_{i}(M-1)\right]$ and $\mathbf{z}_{2}=\left[y_{r}\left(T_{d}\right) y_{i}\left(T_{d}\right) y_{r}\left(T_{d}+1\right) y_{i}\left(T_{d}+1\right) \ldots y_{r}(M+\right.$ 
$\left.\left.T_{d}-1\right) y_{i}\left(M+T_{d}-1\right)\right]$, where $y_{r}(t)$ and $y_{i}(t)$ are the real and imaginary parts of $y(t)$. Due to the circular symmetry assumption, the zero mean random variables $y_{r}(t)$ and $y_{i}(t)$ are independent and identically distributed (i.i.d.). We define $\hat{\rho}_{M L}$ as the maximum likelihood estimate from the vectors $\mathbf{z}_{1}$ and $\mathbf{z}_{2}$. When using the log likelihood ratio test (LLRT), we decide the alternative hypothesis for the present hypothesis test above the threshold, $\eta_{l}$ of the adversary detector. For sufficiently large $M$, we approximate the distribution of the test statistic described in Reference [18] as

$$
\left\{\begin{array}{l}
h_{0}: \hat{\rho}_{M L} \sim \mathcal{N}_{r}\left(0, \frac{1}{2 M}\right) \\
h_{1}: \hat{\rho}_{M L} \sim \mathcal{N}_{r}\left(\rho_{D}, \frac{\left(1-\rho_{D}^{2}\right)^{2}}{2 M}\right) .
\end{array}\right.
$$

This is a classical detection problem with test statistic $\hat{\rho}_{M L}$. To detect and intercept the desired signal, detectors can adopt one of the well-known detection strategies. We consider that the detectors adopt the detection strategy of Neyman-Pearson detection to satisfy a constant false alarm rate constraint [19]. For a Gaussian random variable $r \sim \mathcal{N}_{r}\left(\mu_{r}, \sigma_{r}^{2}\right)$, we have

$$
P\left(r>\eta_{r}\right)=\frac{1}{2} \operatorname{erfc}\left(\frac{\eta_{r}-\mu_{r}}{\sqrt{2} \sigma_{r}}\right),
$$

where $\operatorname{erfc}(\cdot)$ is the complementary error function. Using (14) for $2 M$ real symbols, the required false alarm probability $P_{f a}$ is given by

$$
P_{f a}=P\left(\hat{\rho}_{M L}>\eta_{l} \mid h_{0}\right)=\frac{1}{2} \operatorname{erfc}\left(\sqrt{M} \cdot \eta_{l}\right) .
$$

Thus, the threshold at the detector can be calculated as

$$
\eta_{l}=\frac{1}{\sqrt{M}} \cdot \operatorname{erfc}^{-1}\left(2 P_{f a}\right) .
$$

The detection probability of the FH-OFDMA system is composed of the product of the auto-correlation-based symbol detection probability, that we have analyzed, and the intercept probability of FH patterns from the point of view of an adversary detector, as the two events are independent. Thus, the detection probability, $P_{d}^{F T}$, of the FH-OFDMA system using different FH patterns, such as SBS, cat map, and CSM-based strategies, for $2 M$ real symbols can be obtained using (14), and we get:

$$
P_{d}^{F T}=P_{I}^{F H} \cdot P\left(\hat{\rho}_{M L}>\eta_{l} \mid h_{1}\right)=P_{I}^{F H} \cdot \frac{1}{2} \operatorname{erfc}\left(\sqrt{M} \cdot \frac{\eta_{l}-\rho_{D}}{1-\rho_{D}^{2}}\right),
$$

where $P_{I}^{F H}$ denotes the intercept probability for the FH-OFDMA system using different FH patterns. Based on Reference [13], $P_{I}^{F H}$ has a different value according to the SBS, cat map, CSM-based FH patterns, and FH can, thus, be rewritten by SBS, CAT, and CSM, respectively. Without loss of generality, we assume that the hopping sequence does not change during the $M$ OFDMA symbols, i.e., $2 M$ real symbols. For the SBS FH pattern, the detector is able to intercept the transmitted OFDMA symbol by selecting one cluster from $N_{L}$ clusters, except the previously selected cluster at each period. For $K$ transmitting users $\left(K<N_{L}\right)$, the detector can select one cluster from $K$ clusters at each period. Hence, the $P_{I}^{S B S}$ can be given as

$$
P_{I}^{S B S}= \begin{cases}\frac{2 N_{R}}{N_{L} \times\left(N_{L}-1\right)^{N_{L}-1}}, & K=N_{L}, \\ \frac{2 N_{R}}{K^{N_{L}}}, & K<N_{L} .\end{cases}
$$

For the cat map-based FH pattern, transmitted symbols of each user appear at each symbol period once with mixed order in the time domain. The detector can intercept these 
symbols by selecting one cluster from $K$ clusters at each period and finding the correct order from possible permutations. Hence, the $P_{I}^{C A T}$ can be given by

$$
P_{I}^{C A T}= \begin{cases}\frac{2 N_{R}}{N_{L} \times\left(N_{L}-1\right)^{N_{L}-1} \times N_{L} !}, & K=N_{L}, \\ \frac{2 N_{R}}{K^{N_{L} \times N_{L} !},} & K<N_{L} .\end{cases}
$$

For the CSM-based FH pattern, the detector should select $N_{L}$ clusters from $N_{L} \times K\left(K \leq N_{L}\right)$ symbols and find the order of these symbols after detecting, receiving, and storing the symbols during the period $N_{L}$. Hence, the $P_{I}^{C S M}$ is given by

$$
P_{I}^{C S M}=\frac{2 N_{R}}{N_{L} \times K} P_{N_{L}}, K \leq N_{L} .
$$

Here, ${ }_{n} P_{r}$ denotes the permutation of $n ! /(n-r) !$.

\section{Simulation Results}

The simulation results are provided to analyze whether the CSM-based FH-OFDMA system provides greater LPD capability than the cat map and SBS-based FH-OFDMA systems. We set common parameters of simulation results as the number of subcarriers, $N_{F F T}=220$, excluding guard subcarriers of 36 , number of symbols for cyclic prefix, $T_{c}=T_{d} / 4$, number of clusters as the number of maximum transmitting users, $N_{L}=11$, and $N_{S}=20$. The transmitted data is modulated as QPSK or 16-QAM, and the data is coded by convolutional coding at $1 / 2$ coding rate. The FH-OFDMA signal is sent over a wireless six-ray channel impulse model of the Universal Mobile Telecommunications System-Terrestrial Radio Access System (UTRA) vehicular model A [20]. For the SBS-based FH pattern, we select a Latin squares-based FH pattern of $\alpha=4$. For the cat map-based FH pattern, we consider $\alpha=4, b_{11}=b_{12}=b_{21}=1$, and $b_{22}=2$. For the CSM-based FH pattern, we consider $\alpha=2$, $r_{x}=1, r_{y}=3$, and $K_{C}=3000$.

Before we analyze the detection probabilities of FH-OFDMA systems, we verify that the FH-OFDMA systems based on SBS, cat map, and CSM patterns can achieve frequency diversities by bit error rate (BER) performance. Figure 3 shows the BER results of an OFDMA system without hopping and FH-OFDMA systems based on SBS, cat map, and CSM patterns, in which we adopt two modulation rates as QPSK and 16-QAM over the same channel response. As shown in Figure 3, all the three FH-OFDMA systems can obtain better BER results than the OFDMA system operated without hopping. This result verifies that the SBS, cat map, and CSM-based FH-OFDMA systems can achieve frequency diversities over the channel model. In addition, since the BER results by adopting QPSK are better than those by adopting 16-QAM, we observe that BER performance increases as the modulation rate decreases. Through the simulation results of Figure 3, we show that frequency hopping is a significant and effective method to achieve frequency diversity and improve BER performance in the OFDMA system.

Figure 4 shows the detection probabilities of SBS, cat map, and CSM-based FHOFDMA systems for logarithmic scales as the SNR increases. We set common parameters for the three FH-OFDMA systems as false alarm probability, $P_{f a}=0.05$, redundant number of FH-OFDMA frames, $N_{R}=100$, number of clusters, $N_{L}=11$, and number of transmitting users, $K=11$. As we mentioned previously, since the detection probability of the FHOFDMA system is the product of the symbol detection probability and the intercept probability, the gap among three FH patterns is sustained as constant as SNR increases. As a result, since the CSM-based FH pattern has the lowest intercept probability, the CSM always has a lower detection probability than other FH-OFDMA systems. In addition, we can observe that all the detection probabilities are increased when SNR is varied from -35 to $-15 \mathrm{~dB}$. However, the detection probabilities of the three FH-OFDMA systems converge to a fixed value when the SNR exceeds $-14 \mathrm{~dB}$. 


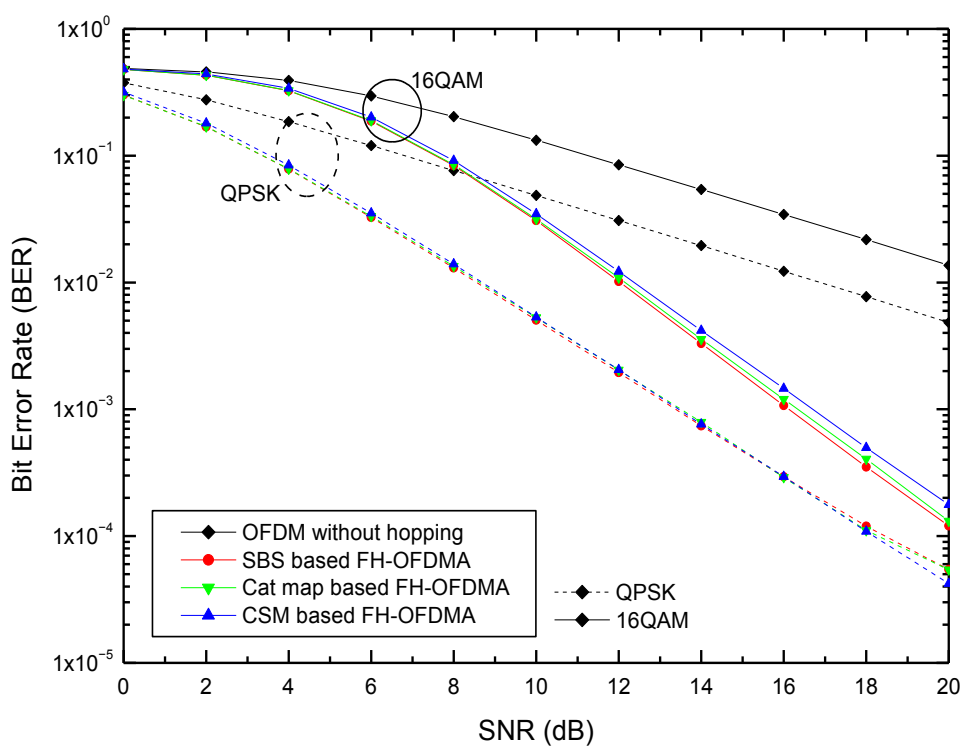

Figure 3. Bit error rate (BER) performances versus signal-to-noise ratio (SNR) compared to SBS, cat map, and CSM-based FH-OFDMA systems.

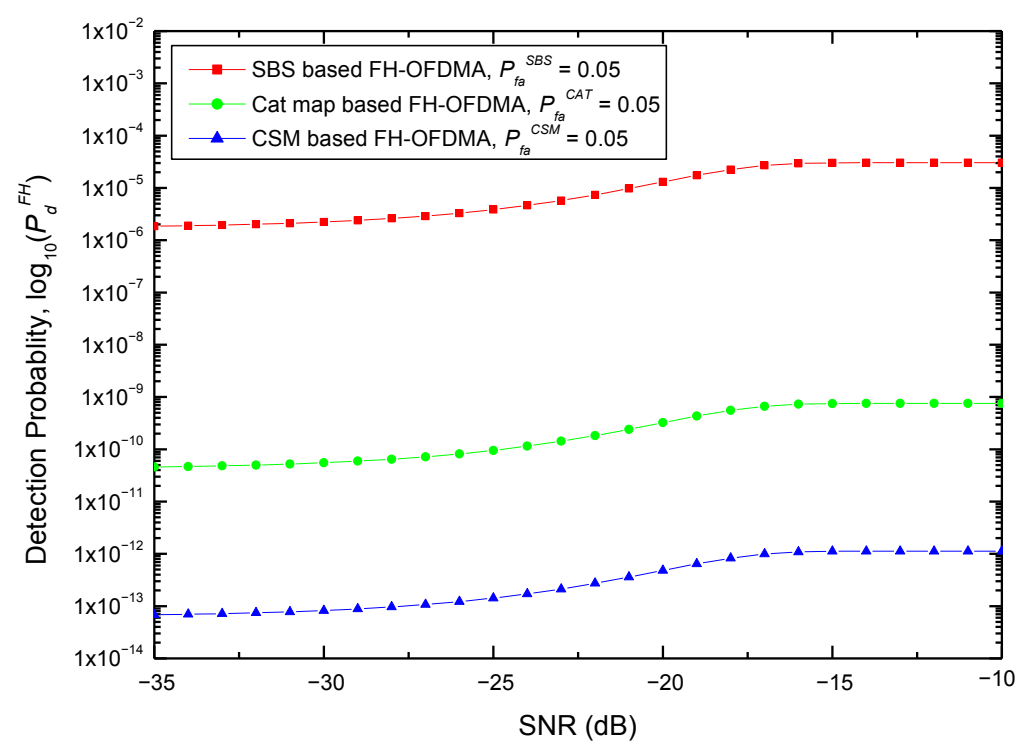

Figure 4. Detection probability versus SNR compared to SBS, cat map, and CSM-based FH-OFDMA systems.

Figure 5 shows the relationship between the detection probability and redundant number of FH-OFDMA frames for detecting for the SBS, cat map, and CSM-based FHOFDMA systems. We set common parameters as false alarm probability, $P_{f a}=0.05$, number of clusters, $N_{L}=11$, number of transmitting users, $K=11$, and SNR $=10 \mathrm{~dB}$. In Figure 5, as the redundant number of FH-OFDMA frames for detecting increases, the detection probability also increases. This result shows that, if a seed number changes after $N_{L}$ periods and the hopping cluster index, thus, changes every FH-OFDMA frame, the LPD capability is enhanced extremely. Figure 5 also shows that the gap among three $\mathrm{FH}$ patterns is sustained as constant regardless of the value of $N_{R}$, since the detection probability of the FH-OFDMA system is the product of the symbol detection probability and the intercept probability. 


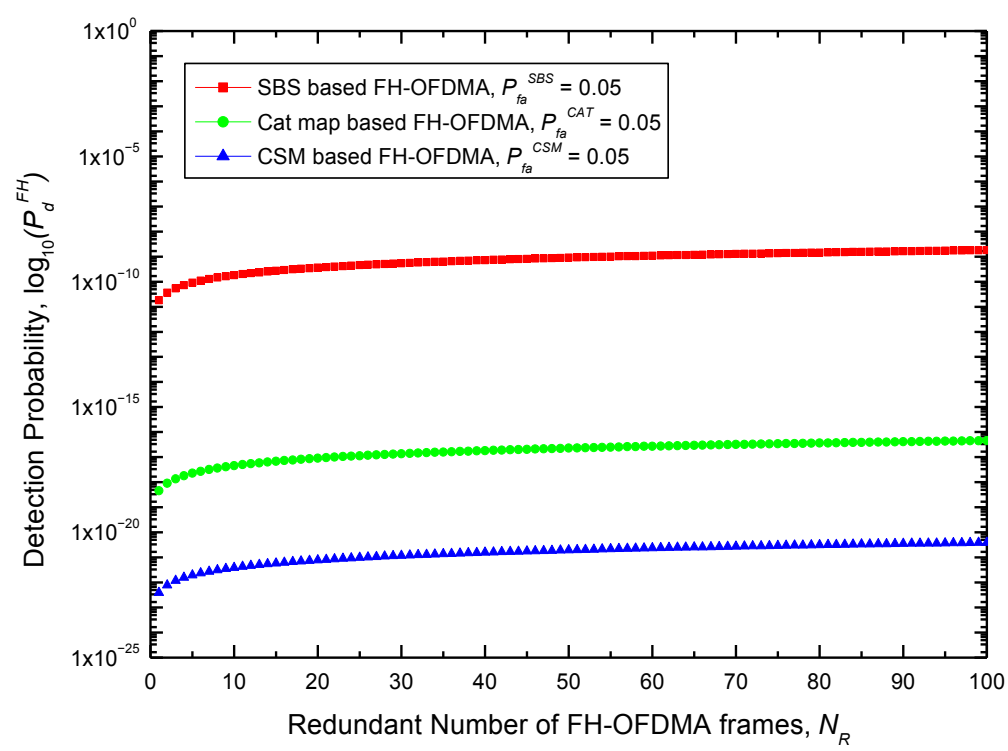

Figure 5. Detection probability versus redundant number of FH-OFDMA frames for detection compared to SBS, cat map, and CSM-based FH-OFDMA systems.

Figure 6 shows the required false alarm probability for detection probabilities for the SBS, cat map, and CSM-based systems. We choose common parameters as SNR $=-20 \mathrm{~dB}$, redundant number of FH-OFDMA frames, $N_{R}=100$, number of clusters, $N_{L}=11$, and number of transmitting users, $K=11$. In Figure 6, we observe that the detection probability at the detector decreases as the required false alarm probability increases. As shown in Figure 6, the CSM always has a lower detection probability than the cat map and SBS for all values of the false alarm probabilities.

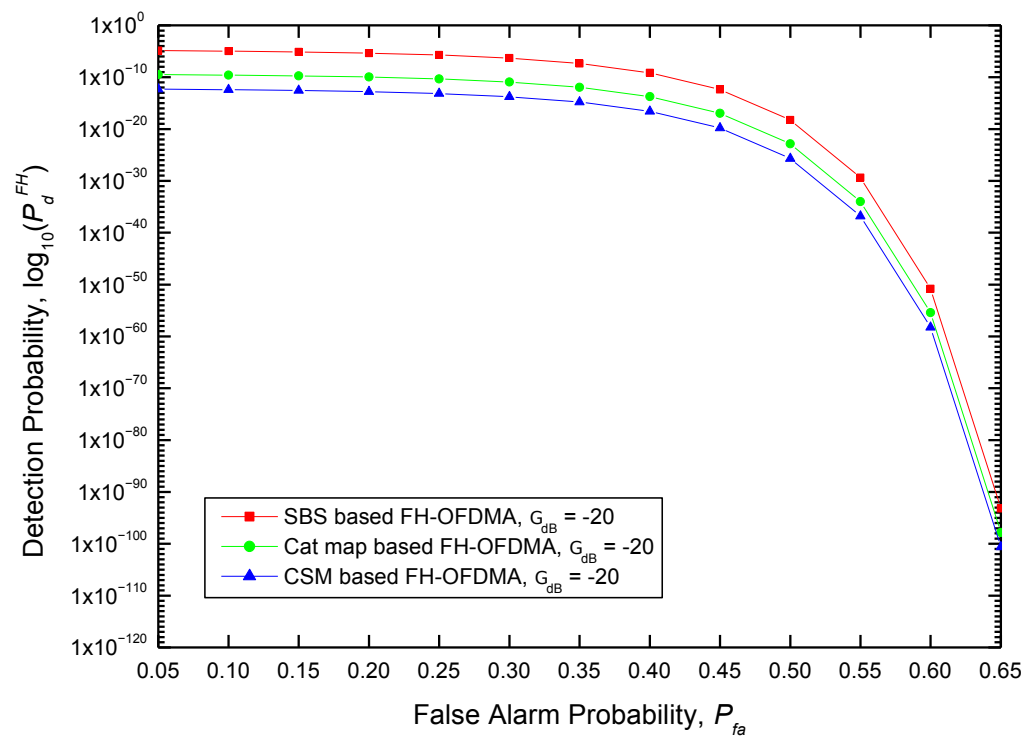

Figure 6. Required false alarm probability versus detection probability compared to SBS, cat map, and CSM-based FH-OFDMA systems.

Figure 7 shows the detection probabilities of the SBS, cat map, and CSM-based FHOFDMA systems for logarithmic scales as the user load increases from 0.1 to 1.0. The user load is defined as the ratio of the number of transmitting users $K$ to the number of clusters $N_{L}$. We set the parameters as false alarm probability, $P_{f a}=0.05$, redundant number of FH-OFDMA frames, $N_{R}=100$, number of clusters, $N_{L}=11$, and SNR $=-20 \mathrm{~dB}$. When $K=1$, the detection probabilities of the cat map and CSM-based systems have the same value and lower than that of the SBS-based system. When $K$ increases above 1 , the detection 
probability of the CSM becomes smaller than that of the cat map. As shown in Figure 7, we can also observe that when user load approaches 1 , the detection probabilities are decreased. This means that an increase in user load enhances the LPD capability of the FH-OFDMA system.

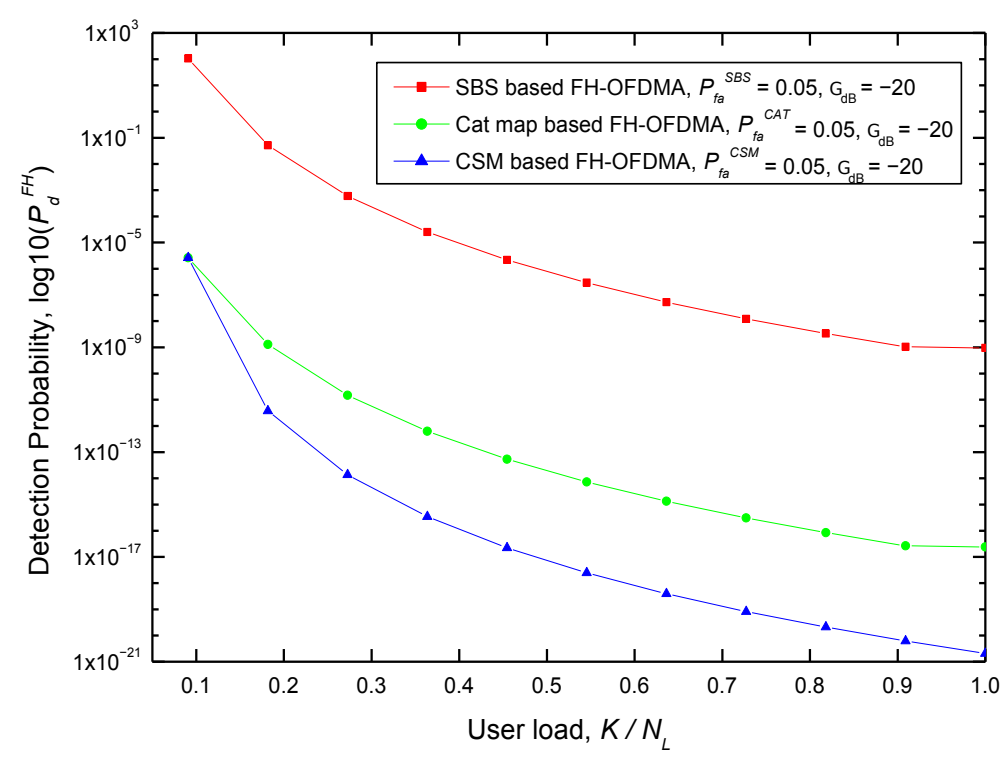

Figure 7. Detection probability versus user load compared to SBS, cat map, and CSM-based FH patterns.

From Figures 4-7, we conclude that the CSM-based FH-OFDMA system has the greatest LPD capability. Figure 8 shows the effect of the required false alarm probability, $P_{f a}$ of the CSM-based FH-OFDMA system. We chose three values of $P_{f a}$ as 0.05, 0.1, and 0.2 , while setting redundant number of FH-OFDMA frames, $N_{R}=100$, number of clusters, $N_{L}=11$, and number of transmitting users, $K=11$. For the results, we varied the SNR from -35 to $-10 \mathrm{~dB}$. As the SNR increases from -35 to $-15 \mathrm{~dB}$, detection probabilities of the CSM sharply increase for all values of $P_{f a}$. Above SNR $=-15 \mathrm{~dB}$, the detection probabilities are stable. As seen in Figure 8, as the required false alarm probability increases, the detection probability decreases because the threshold to detect the FH-OFDMA signal increases.

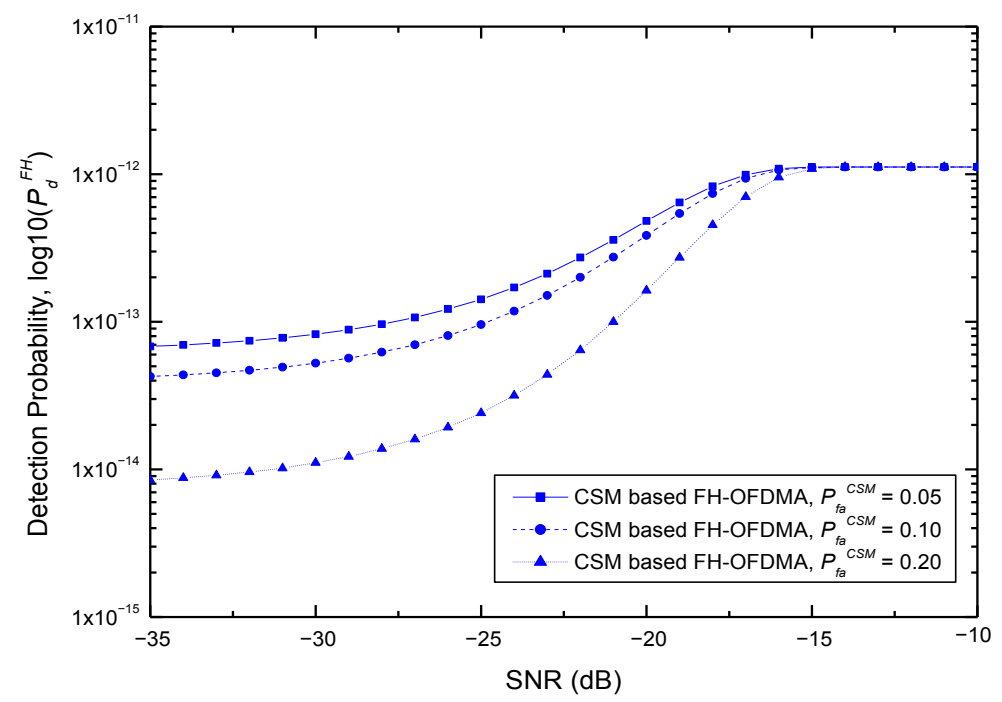

Figure 8. SNR versus detection probability compared to required false alarm probabilities of $0.05,0.1$, and 0.2 for CSM-based FH-OFDMA system. 


\section{Conclusions}

In this study, we analyzed the detection probability of the CSM-based FH-OFDMA system and compared it with the cat map and SBS-based FH-OFDMA systems. We theoretically derived the LPD capability of the CSM-based FH-OFDMA system using autocorrelation-based spectrum sensing technique with hypothesis testing, and we showed the CSM-based system has greater LPD capability than the cat map and SBS-based systems. Simulation results also show the CSM-based system has similar BER performance as the two systems, but the CSM-based system decreases the detection probability dramatically in various simulation environments. As the redundant number of detecting frames or SNR increases, and the number of simultaneous users decreases, an intentional eavesdropper has a higher detection probability to find FH patterns, and the CSM stands out among the three FH patterns. Additionally, we found that the gap among the three FH patterns is sustained as the redundant number of frames or SNR increases, since the detection probability of the FH-OFDMA system is the product of the symbol detection probability and the intercept probability. The CSM-based FH pattern can be applied to time-frequency hopping domain, as well as the FH-OFDMA system. The CSM-based FH pattern has a limit to having symbol buffers of cluster size. Even considering the limitation, the CSM-based FH pattern has powerful LPD capability because the time and frequency transmitting sequences are mixed.

Author Contributions: Conceptualization, J.J. and J.L.; methodology, J.J. and J.L.; software, J.J. and S.P.; validation, J.L., H.K., and S.K.; investigation, J.J.; writing-original-draft preparation, J.J. and S.P.;writing-review and editing, J.J and S.P. All authors have read and agreed to the published version of the manuscript.

Funding: This research received no external funding.

Institutional Review Board Statement: Not applicable.

Informed Consent Statement: Not applicable.

Data Availability Statement: Not applicable.

Conflicts of Interest: The authors declare no conflict of interest.

\section{References}

1. Stamatiou, K.; Proakis, J.G. Performance analysis of a coherent FH-MA system based on Latin squares. IEEE Trans. Wirel. Commun. 2007, 6, 4183-4192. [CrossRef]

2. Jung, J.; Lim, J.; Kang, H.; Park, H. Multiple access interference-free multichannel slotted aloha scheme for time-asynchronous OFDMA systems. IEICE Trans. Commun. 2015, E98-B, 686-700. [CrossRef]

3. Jung, J.; Lim, J. Group contention-based OFDMA MAC protocol for multiple access interference-free in WLAN systems. IEEE Trans. Wirel. Commun. 2012, 11, 648-658. [CrossRef]

4. Verhulst, D.; Mouly, M.; Szpirglas, J. Slow frequency hopping multiple access for digital cellular radiotelephone. IEEE J. Sel. Areas Commun. 1984, 2, 563-574. [CrossRef]

5. Olofsson, H.; Naslund, J.; Skold, J. Interference diversity gain in frequency hopping GSM. In Proceedings of the IEEE 45th Vehicle Technology Conference (VTC), Chicago, IL, USA, 25-28 July 1995.

6. Chiani, M.; Conti, A.; Andrisano, O. Outage evaluation for slow frequency-hopping mobile radio systems. IEEE Trans. Commun 1999, 47, 1865-1874. [CrossRef]

7. Lee, H.; Ahn, J.; Kim, Y.; Chung, J. Outage evaluation for slow frequency-hopping mobile radio systems. Appl. Sci. 2020, 10, 1733. [CrossRef]

8. Stamatiou, K.; Proakis, J.G. A performance analysis of coded frequency-hopped OFDMA. In Proceedings of the IEEE Wireless Communications and Networking Conference (WCNC), New Orleans, LA, USA, 13-17 March 2005.

9. Wang, C.; Zhang, X.; Yang, D. Evaluation of Welch-Costas frequency hopping pattern for OFDM cellular system. In Proceedings of the IEEE 18th International Symposium on Personal, Indoor and Mobile Radio Communications (PIMRC), Athens, Greece, 3-7 September 2007.

10. Lightfoot, L.; Zhang, L.; Ren, J.; Li, T. Secure collision-free frequency hopping for OFDMA-based wireless networks. EURASIP J Adv. Signal. Proc. 2009, 2009, 361063. [CrossRef]

11. Zhu, D.; Natarajan, B. Residue number system arithmetic assisted coded frequency-hopped OFDMA. EURASIP J. Wirel. Commun. Netw. 2009, 2009, 263695. [CrossRef] 
12. Li, P.; Fan, C.; Yang, Y.; Wang, Y. New bounds on wide-gap frequency-hopping sequences. IEEE Commun. Lett. 2019, 23, 1050-1053. [CrossRef]

13. Jung, J.; Lim, J. Chaotic standard map based frequency hopping OFDMA for low probability of intercept. IEEE Commun. Lett. 2011, 15, 1019-1021. [CrossRef]

14. Axell, E.; Leus, G.; Larsson, E.G. Overview of spectrum sensing for cognitive radio. In Proceedings of the 2nd International Workshop on Cognitive Information Processing (CIP), Elba, Italy, 14-16 June 2010.

15. Ning, B.; Li, Z.; Guan, L.; Zhou, F. Probabilistic frequency-hopping sequence with low probability of detection based on spectrum sensing. IET Commun. 2017, 11, 2147-2153. [CrossRef]

16. Lee, K.G.; Oh, S.J. Detection of fast frequency-hopping signals using dirty template in the frequency domain. IEEE Wirel. Comm. Letters 2019, 8, 281-284. [CrossRef]

17. Li, Z.; Liu, R.; Lin, X.; Shi, H. Detection of frequency-hopping signals based on deep neural networks. In Proceedings of the IEEE 3rd International Conference on Communication and Information Systems (ICCIS), Singapore, 28-30 December 2018.

18. Chaudhari, S.; Koivunen, V.; Poor, H.V. Autocorrelation-based decentralized sequential detection of OFDM signals in cognitive radios. IEEE Trans. Signal. Process 2009, 57, 2690-2700. [CrossRef]

19. Barkat, M. Signal and Detection and Estimation, 2nd ed.; Artech House: Boston, MA, USA, 2005.

20. UMTS. Selection Procedures for Choice of Radio Transmission Technologies of UMTS (TR 101 112, UMTS 30.03, V3.1.0 (1997-11)); 3GPP Mobile Competence Centre: Sophia Antipolis, France, 1997. 